\title{
The Postoperative Course of Choroidal and Central Retinal Thickness in Epiretinal Membranes with Respect to Membrane Severity
}

\author{
Gergely Zobor ${ }^{a, b}$ Stefan Sacu ${ }^{a, b}$ Marlene Hollaus ${ }^{a, b}$ Leyla Aliyeva ${ }^{b}$ \\ Alexandra-Stephanie Schmalek ${ }^{a, b}$ Ursula Schmidt-Erfurth ${ }^{a}$ \\ Michael Georgopoulos ${ }^{a}$
}

aDepartment of Ophthalmology and Optometry, Medical University of Vienna, Vienna, Austria; bienna Clinical Trial

Center, Medical University of Vienna, Vienna, Austria

\section{Keywords}

Epiretinal membrane $\cdot$ Membrane peeling $\cdot$ Choroidal

thickness · Retinal thickness · Visual acuity

\begin{abstract}
Introduction: The aim of this study was to evaluate the relation between choroidal thickness (CT), central retinal thickness (CRT) and best-corrected visual acuity (BCVA) after surgery for idiopathic epiretinal membrane (iERM). Methods: Patients with 4 severity stages of iERM, who underwent vitrectomy with membrane- and internal limiting membrane peeling, were included in this prospective study. CRT, CT, and BCVA were assessed at baseline (BSL), 1 week, 1 and 3 months postoperatively. Results: Twenty-one eyes were phakic, 11 eyes pseudophakic at BSL, in 14 cases combined cataract surgery was performed. BCVA was highest in stage 1 and 2 , lowest in stage 4 iERM $(p<0.001)$ and correlated with CRT. After surgery, CRT decreased and BCVA increased significantly $(p<0.05)$. CT did not show significant differences among stages ( $p=0.23$ ). BSL CRT did not differ between phakic and pseudophakic eyes, the least reduction after surgery
\end{abstract}

karger@karger.com www.karger.com/ore

Karger $\stackrel{\text { ' }}{5}$
(C) 2021 The Author(s)

Published by S. Karger AG, Basel

This is an Open Access article licensed under the Creative Common Attribution-NonCommercial-4.0 International License (CC BY-NC) (http://www.karger.com/Services/OpenAccessLicense), applicable to the online version of the article only. Usage and distribution for commercial purposes requires written permission. was detected in patients who underwent combined cataract surgery and vitrectomy. BSL CT was greater in phakic than in pseudophakic eyes ( $p=0.033$ ). Postoperative CT decreased in pseudophakic and phakic eyes, but remained higher after combined surgery ( $p=0.0048)$. Conclusion: $C T$ is not related to the severity of iERM. Choroidal changes did not influence the BCVA. Additional cataract surgery seems to cause longer recovery in CT and CRT.

(c) 2021 The Author(s)

Published by S. Karger AG, Basel

\section{Introduction}

Epiretinal membrane (ERM), also known as macular pucker, is a condition caused by nonvascular fibrocellular proliferation that develops on the surface of the internal limiting membrane (ILM). Its pathogenic mechanism has an unknown etiology and can be idiopathic or secondary due to other ocular diseases, trauma, or previous intraocular surgery. The incidence of idiopathic ERM (iERM) ranges from $2 \%$ to $20 \%$, increasing with age of patients [1-4]. It results in a tangential traction, causing retinal 
changes, such as retinal layers' thickening, surface wrinkling, and/or nerve fiber layer fibrillation, which cause distortion and reduction of vision.

The standard therapy of macular pucker is vitrectomy combined with dye assisted ILM peeling [3]. Previous studies have shown that good visual outcomes after ERM peeling are dependent on an intact photoreceptor inner segment/outer segment junction, as seen in preoperative spectral-domain optical coherence tomography (OCT) $[3,4]$. Unfortunately, photoreceptor defects and increased retinal thickness in the fovea persist in some cases despite successful surgery. These findings contribute to the fact that some patients do not recover complete visual function after vitrectomy for ERMs. There are also patients whose visual function does not improve after ERM surgery that show a normal appearing photoreceptor layer on spectral-domain $\mathrm{OCT}$, indicating that other factors might contribute to the remaining reduction in visual function after surgery [3-5].

The choroid is a vascular layer between the inner border of the sclera and the outer border of the retinal epithelium that supplies the retina with oxygen and nutrients and is furthermore responsible for its thermal regulation. It is believed to be the only nourishment to the foveal avascular zone. Therefore, diseases localized in the fovea that are associated with alterations in retinal thickness may influence the choroidal blood flow and in consequence, choroidal thickness (CT). Recent studies suggest that mechanical traction and ischemia might have an effect on the choroid in the presence of iERM. The traction on the retina exerted by the iERM causes an increase in CT and affects retinal vessels. Retinal arteries become dilated and tortuous, which may alter the hemodynamics of the affected area $[6,7]$. It was observed that the CT and the blood flow area of choriocapillaris were reduced in patients with iERM. After vitrectomy, the blood flow area of choriocapillaris increased, while CT showed inconsistent results over previous studies [8-11].

Structural changes and the role of the choroid with regard to ERM severity stages or vitrectomy combined with cataract surgery versus vitrectomy without cataract surgery have not yet been explained sufficiently $[8,9]$. With the help of new OCT modalities like enhanced depth imaging, swept-source OCT (SS-OCT) imaging and OCT angiography, a better pre- and postoperative visualization and evaluation of retinal structures and the choroid became possible [12]. Hence, using novel OCT technologies, this work aims to study choroidal and retinal changes in patients with iERM before and after vitreomacular surgery and to examine its correlation to anatomical changes within visual function.

Influence of ERM Surgery on Retinal and Choroidal Structures

\section{Patients and Methods}

This was a prospective, open, nonrandomized, observational study performed at the Department of Ophthalmology, Medical University of Vienna. The study included patients affected by idiopathic ERMs for which 23-gauge pars plana vitrectomy with membrane peeling was performed. Exclusion criteria were coexisting diabetes, any vascular disease or age-related macular degeneration, glaucoma, high myopia, or hyperopia. This study was conducted in adherence to the Declaration of Helsinki and the Institutional Ethics Committee approved the study (www.clinicaltrials. gov, NCT02617147). All patients gave informed consent.

In all cases, 23-gauge 3-port pars plana vitrectomy was performed using an Örtli machine (Oertli Instruments, Berneck, Switzerland) with a widefield viewing system (MiniQuad XL VIT contact lens; Volk, Mentor, OH, USA) or a biom (Oculus Surgical Inc., Port St. Lucie, FL, USA). Following vitreous body removal, ERM and ILM were removed using a microforceps, after which the ILM was stained using viewILM dye (Alchimia, Ponte San Nicola, PD, Italy). During peeling, a contact lens (Landers contact lens; Ocular Instruments Inc., Bellevue, WA, USA) was used for visualization of the fovea in all cases. Criteria for additional cataract surgery included any opalescence causing a decrease in visualization of the retina or LOCS grading exceeding $\mathrm{NO}>2, \mathrm{NC}>2, \mathrm{C}>1$ or $\mathrm{PSC}>0.5$.

Examinations were performed within 2 weeks before surgery (baseline [BSL]), 1 week, 1 month, and 3 months (M3) after surgery. At each visit, a detailed ophthalmological examination was carried out including best-corrected visual acuity (BCVA) and SSOCT images measuring retinal and choroidal architecture. The fellow eyes were evaluated as the control group.

OCT images were performed with the SS-OCT device (DRIAtlantis; Topcon, Tokyo, Japan) at each visit. In all cases, we performed a single line scan with a resolution of $1 \mathrm{~mm}$, with a length of $12 \mathrm{~mm}$. Central retinal thickness (CRT) was measured manually as the distance between the ILM and the retinal pigment epithelium (RPE). CT was measured manually on blackand-white images between the line representing RPE (the outermost hyper-reflective retinal layer) and the outer choroidoscleral boundary (the outer hyper-reflective line of the choroid). For the measurement of the central choroidal volume (CV), images were analyzed with OCTAVO, a validated image analysis software which was developed at the Vienna Reading Center (Department of Ophthalmology, Medical University of Vienna, Austria) $[13,14]$. Images were positioned manually, and an ETDRS grid was centered to the fovea. With the help of this grid, the central $1 \mathrm{~mm}^{2}$ area was defined and the volume of the choroid was measured between the RPE and the outer choroidoscleral boundary in $\mathrm{mm}^{3}$ (Fig. 1).

Study eyes were categorized according to the ERM staging scheme of Govetto $[15,16]$ in 4 groups:

- Stage 1 was defined as the presence of a mild ERM with negligible morphologic or anatomic disruption. All retinal layers and the foveal depression were clearly detectable.

- Stage 2 was defined as the presence of ERMs associated with more progressive retinal distortion. While the foveal depression was lost and a characteristic stretching of the outer nuclear layer was present, all retinal layers were clearly identified with OCT.

- Stage 3 was defined as the presence of an ERM with continuous ectopic inner foveal layers anomalously crossing the central fo-

Ophthalmic Res 2021;64:1020-1028

1021 


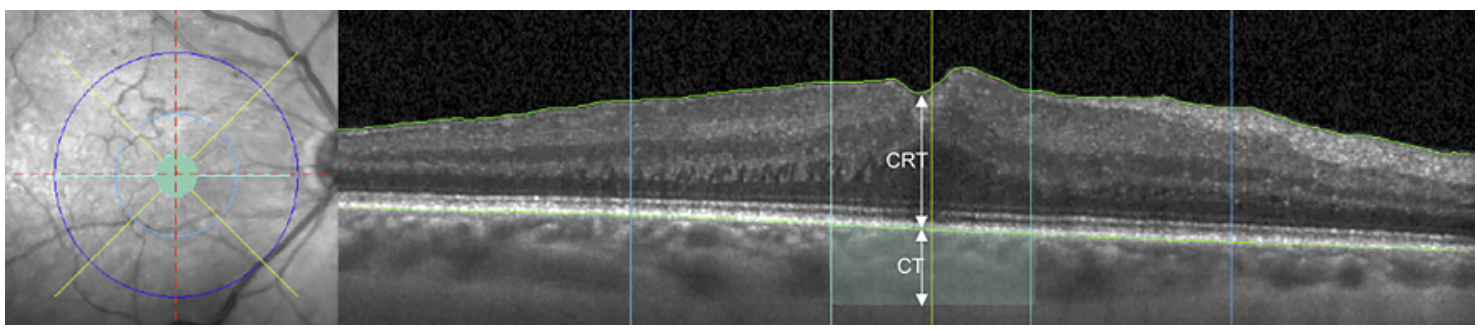

Fig. 1. Measurement of CRT, CT in the fovea and the central CV using the OCTAVO software. Images were positioned manually and an ETDRS grid was centered to the fovea. With the help of this grid, the central $1 \mathrm{~mm}^{2}$ area was defined (bright blue circle and lines) and the volume of the choroid (green shaded area) was measured underneath between the RPE and the outer choroidoscleral boundary in $\mathrm{mm}^{3}$. CT, choroidal thickness; CV, choroidal volume; CRT, central retinal thickness; RPE, retinal pigment epithelium.

Table 1. BSL characteristics of patients depending on the ERM stages

\begin{tabular}{lllllll}
\hline & Stage $1(n=4)$ & Stage $2(n=14)$ & Stage $3(n=11)$ & Stage 4 $(n=3)$ & All stages $(n=32)$ & Control $(n=22)$ \\
\hline Age, years & $69 \pm 7.1$ & $73 \pm 5.7$ & $70.9 \pm 6.9$ & $66 \pm 4.3$ & $71.1 \pm 6.3$ & $69.7 \pm 6.5$ \\
BCVA, logMAR & $0.12 \pm 0.25$ & $0.19 \pm 0.12$ & $0.27 \pm 0.21^{* *}$ & $0.63 \pm 0.05^{* * *}$ & $0.25 \pm 0.21^{* *}$ & $0.1 \pm 0.22$ \\
CRT, $\mu$ m & $359.7 \pm 55.1^{* *}$ & $428.9 \pm 57.7^{* * *}$ & $489.5 \pm 66.8^{* * *}$ & $619 \pm 57.9^{* * *}$ & $453.7 \pm 84^{* * *}$ & $284.4 \pm 20$ \\
Central CT, $\mu \mathrm{m}$ & $246.6 \pm 42.1$ & $213.5 \pm 48.4$ & $267.4 \pm 85.3$ & $242.6 \pm 33.8$ & $238.7 \pm 64.6$ & $220.9 \pm 65.3$ \\
Central CV, $\mathrm{mm}^{3}$ & $0.219 \pm 0.03$ & $0.183 \pm 0.03$ & $0.217 \pm 0.04$ & $0.22 \pm 0.03$ & $0.202 \pm 0.04$ & $0.197 \pm 0.04$ \\
\hline
\end{tabular}

BCVA, best-corrected visual acuity; CRT, central retinal thickness; $C T$, choroidal thickness; CV, choroidal volume. Significantly relevant differences in comparison to control values are marked: ${ }^{*} p<0.05 ; * * p<0.01,{ }^{* * *} p<0.001$.

Table 2. Postoperative results at $M 3$ depending on the ERM stages

\begin{tabular}{llllll}
\hline & Stage $1(n=4)$ & Stage $2(n=14)$ & Stage $3(n=11)$ & Stage 4 $(n=3)$ & All stages $(n=32)$ \\
\hline BCVA, logMAR & $0.1 \pm 0.17$ & $0.19 \pm 0.21$ & $0.13 \pm 0.13$ & $0.4 \pm 0.16^{* *}$ & $0.18 \pm 0.01^{*}$ \\
CRT, $\mu \mathrm{m}$ & $423.7 \pm 52.1$ & $396.5 \pm 62.4$ & $426.6 \pm 52.6^{* *}$ & $483 \pm 5.9^{* *}$ & $416.3 \pm 58.3^{*}$ \\
Central CT, $\mu \mathrm{m}$ & $245.6 \pm 52.6$ & $207.3 \pm 47.1$ & $222.4 \pm 78.8^{*}$ & $233.8 \pm 31.8$ & $219.7 \pm 58.7$ \\
Central CV, $\mathrm{mm}^{3}$ & $0.218 \pm 0.02$ & $0.184 \pm 0.04$ & $0.217 \pm 0.047$ & $0.255 \pm 0.034$ & $0.206 \pm 0.045$ \\
\hline
\end{tabular}

BCVA, best-corrected visual acuity; CRT, central retinal thickness; CT, choroidal thickness; CV, choroidal volume; $B S L$, baseline; $M 3$, month 3 . Significantly relevant changes in comparison to BSL results of each stage are marked: ${ }^{*} p<0.05$; ${ }^{* *} p<0.01,{ }^{* * *} p<0.001$.

veal area. The foveal depression was absent and widening of the outer nuclear layer was often less pronounced.

- Stage 4 was defined as an ERM complicated by significant retinal thickening and remarkable anatomic disruption of the macula. Retinal layers were noted to be significantly distorted, disorganized, and not clearly identified with OCT.

The patients were also categorized and the results analyzed according to lens status and performed surgical procedure. The statistical analysis of the data was conducted by using the JMP 14 statistical software (SAS Institute Cary, Cary, NC, USA). Normality of data was assessed by evaluation of the histogram plots. If appropriate, correlation parameters were calculated using Pearson or Spearman analysis. If parametric analysis was possible, Student's $t$ test for paired data was performed for all parametric comparisons between preoperative and postoperative examinations. If parametric analysis was not possible, Wilcoxon test was applied to assess the significance of differences between preoperative and postoperative data. The same level of significance $(p<0.05)$ was used in all cases.

\section{Results}

Thirty-two patients (15 female, 17 male) with a mean age of 71 years (range between 60 and 85 years) were included in the study. Regarding the lens status, 21 eyes were phakic and 11 eyes were pseudophakic at the begin- 


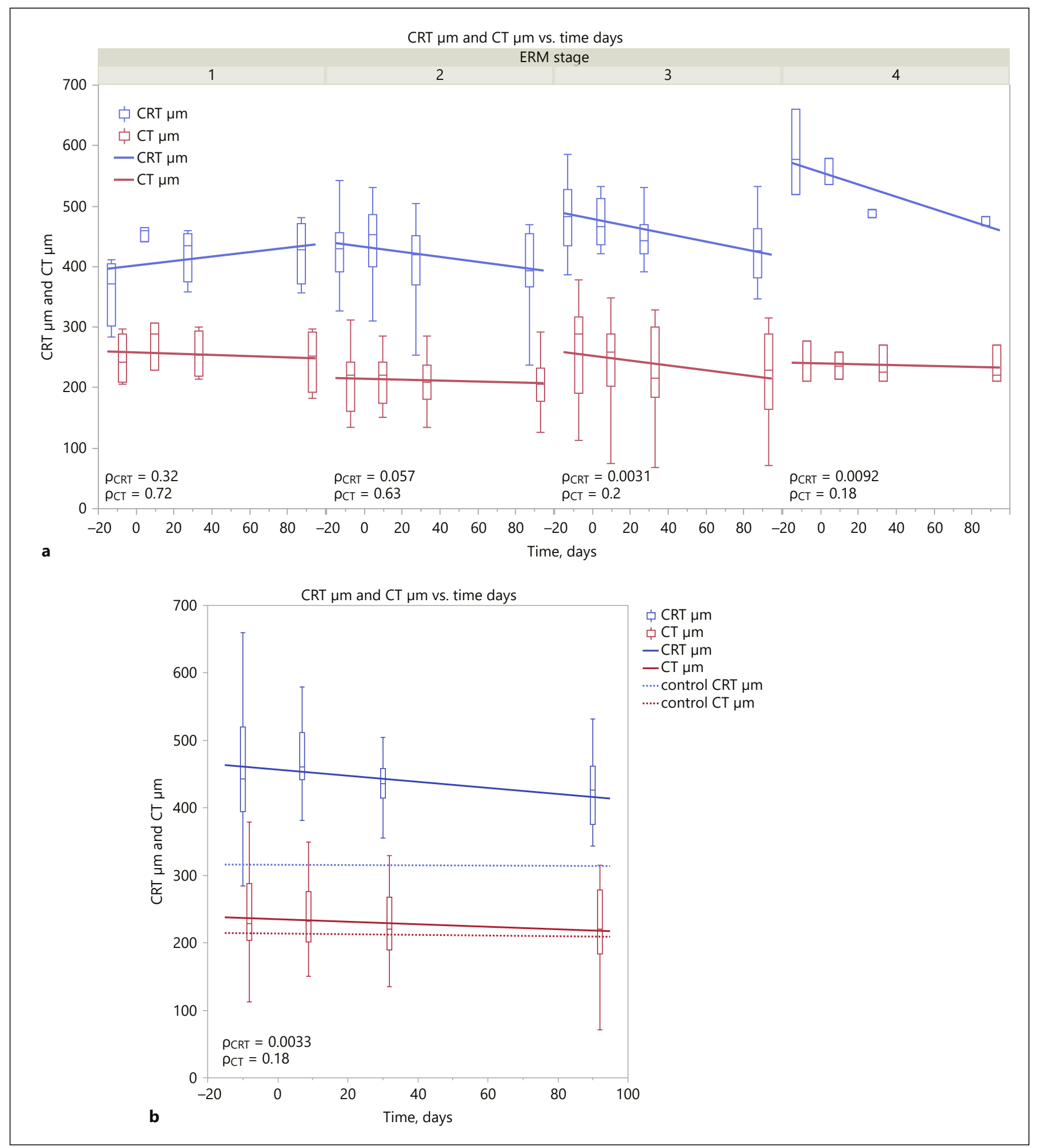

Fig. 2. Changes of the CRT (blue) and CT (red) over time (day $-10=\mathrm{BSL}$; day $0=$ day of surgery; day $7=\mathrm{W} 1$, day $30=\mathrm{M} 1$ and day $90=$ M3; respectively). a The time course depending on ERM stages. b The average changes of the parameters over time. The largest improvement of CRT was observed in stage 3 and 4 ERMs, while the largest improvement of CT was observed in stage 3 ERMs. For comparison, CRT and CT values of control eyes are shown with dashed blue and red lines on $\mathbf{b}$, respectively. CT, choroidal thickness; CRT, central retinal thickness; W1, week 1; M1, month 1 ; M3, month 3 . 
Fig. 3. Changes of the CRT (blue) and CT (red) after surgery at week 1 (W1), month 1 (M1), and month 3 (M3). Patients were categorized depending on the performed surgical procedure $(1=$ vitrectomy, $2=$ phacoemulsification combined with vitrectomy). CT, choroidal thickness; CRT, central retinal thickness; W1, week 1; M1, month 1 ; M3, month 3.

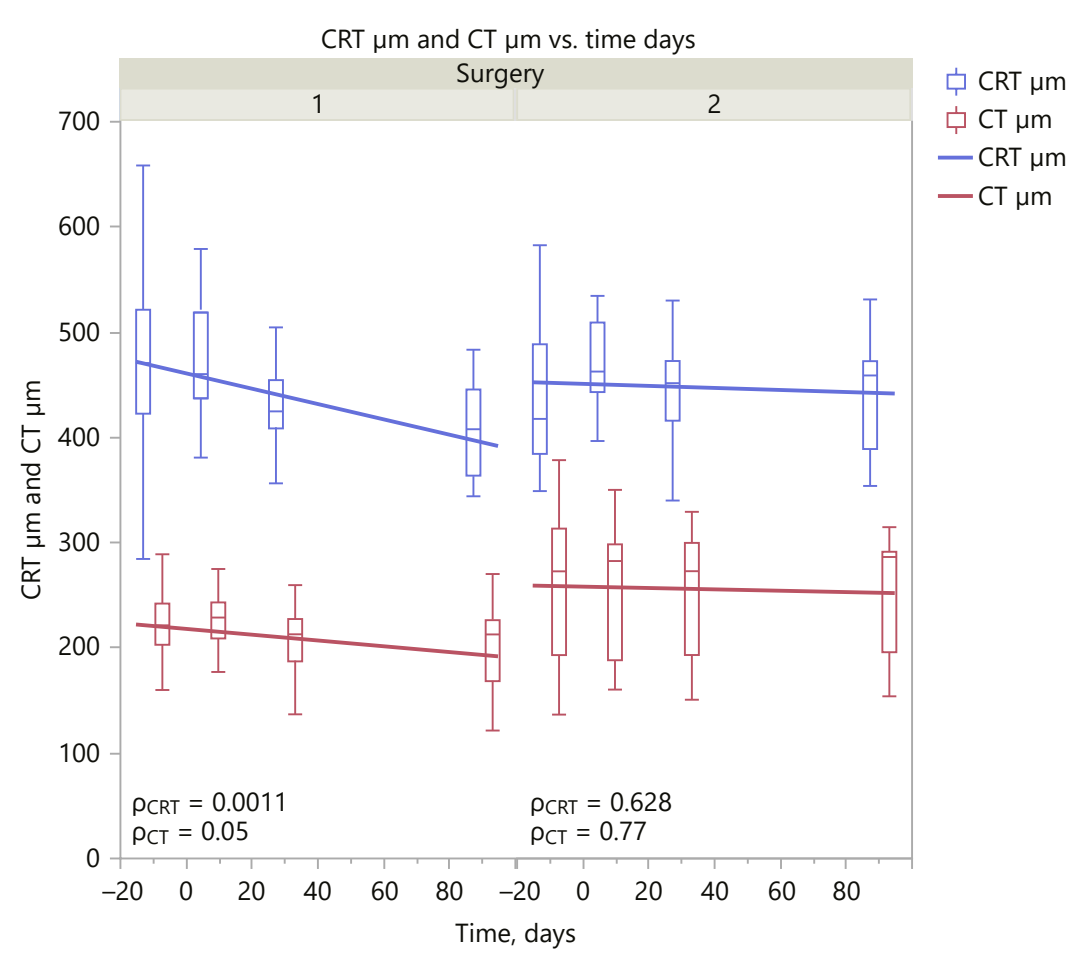

ning of the study. In 14 out of the 21 phakic eyes, combined phacoemulsification and vitrectomy were performed ( $n=14)$, the remaining 7 phakic and 11 pseudophakic patients underwent solely vitrectomy $(n=18)$. Ten patients were diagnosed with ERM on the contralateral eye as well; these results were excluded from the control group.

BSL characteristics of patients depending on ERM stage are listed in Table 1. BCVA was significantly higher in stage 1 and stage 2 and was lowest in stage $4(p<0.001)$. In general, BCVA showed a moderate correlation with CRT $\left(r_{2}=0.59, p<0.001\right)$, the latter being the lowest in eyes with stage 1 ERMs and the highest in eyes with stage 4 ERMs. The thickness of the underlying choroid did not show significant differences among the groups or in comparison to the control eyes at BSL $\left(\mathrm{CT}_{\mathrm{All}}\right.$ vs. $\mathrm{CT}_{\mathrm{Control}}$ was $p=0.23$ ). A correlation between BCVA and CT at BSL was not detected. In line with these findings, the central $\mathrm{CV}$ did not differ among the groups or in comparison to the control group, in which CV was $0.197 \pm 0.04 \mathrm{~mm}^{3}$. There is some evidence in the literature that total CT diminishes with age [17], which could explain the somewhat thinner choroid in stage 2 ERMs, where average age of patients was highest.
After successful surgery, postoperative results showed a reduction of CRT and improvement of BCVA in all cases $(p<0.05)$. The highest improvements of CRT and BCVA were observed in stage 3 and 4 ERMs $(p<0.01$ and $p<0.05$, respectively). Choroidal values did not show a significant reduction over the course of time and a correlation between BCVA and CT was not observed postoperatively (Table 2; Fig. 2).

Interestingly, CT values showed a marked difference depending on the lens status. CT was significantly higher in phakic eyes $(n=21)$ than in pseudophakic eyes $(n=11)$ at BSL $(p=0.033)$. After vitrectomy, CT values showed a reduction in pseudophakic eyes $(n=11)$ and in phakic eyes undergoing vitrectomy $(n=7)$. In contrast, CT values remained relatively unchanged in cases where combined phacoemulsification and vitrectomy were performed $(n=$ 14 ; CT was $261 \pm 77.1 \mu \mathrm{m}$ at BSL and $253.3 \pm 53.6 \mu \mathrm{m}$ at M3, $p=0.2$ ) but also markedly higher over time in comparison to cases in which solely vitrectomy was performed $(n=18)$.

CRT values did not differ significantly at BSL between phakic and pseudophakic eyes (449.4 $\pm 71.5 \mu \mathrm{m}$ and 462.8 $\pm 110.3 \mu \mathrm{m}$, respectively, $p=0.5)$. In the postoperative follow-up, the least reduction of retinal thickness was detected in patients who underwent combined cataract sur- 
Table 3. BSL characteristics of patients depending on the lens status before surgery
Table 4. M3 results characteristics of patients depending on the lens status before surgery and the performed surgical procedure

\begin{tabular}{llll}
\hline BSL & Phakic $(n=21)$ & & $\begin{array}{l}\text { Pseudophakic } \\
(n=11)\end{array}$ \\
\cline { 2 - 3 } & vitrectomy $(n=7)$ & $\begin{array}{l}\text { combined surgery } \\
(n=14)\end{array}$ & $0.25 \pm 0.07$ \\
\hline BCVA, logMAR & $\begin{array}{l}0.2 \pm 0.17 \\
0.18 \pm 0.17\end{array}$ & $0.21 \pm 0.18$ & $462.8 \pm 110.4$ \\
\hline CRT, $\mu \mathrm{m}$ & $\begin{array}{l}449.5 \pm 71.5 \\
470.3 \pm 60.9\end{array}$ & $439.07 \pm 76.1$ & $212.05 \pm 50.6$ \\
\hline Central CT, $\mu \mathrm{m}$ & $252.7 \pm 67.7^{*}$ & $261 \pm 77.1^{*}$ & $0.201 \pm 0.03$ \\
\hline Central CV, $\mathrm{mm}{ }^{3}$ & $236.2 \pm 44.2$ & & \\
\hline
\end{tabular}

Phakic patients were further divided into 2 subgroups depending on the planned surgical performance. CT was significantly greater in phakic eyes in comparison to pseudophakic eyes at BSL. BCVA, best-corrected visual acuity; CRT, central retinal thickness; CT, choroidal thickness; CV, choroidal volume; BSL, baseline. Significantly relevant differences between phakic and pseudophakic eyes are marked: ${ }^{*} p<0.05$; $^{* *} p<0.01$, ${ }^{* * *} p<0.001$.

\begin{tabular}{lllll}
\hline M3 & $\begin{array}{l}\text { Phakic, } \\
\text { vitrectomy } \\
(n=7)\end{array}$ & $\begin{array}{l}\text { Combined } \\
\text { surgery } \\
(n=14)\end{array}$ & $\begin{array}{l}\text { Pseudophakic, } \\
\text { vitrectomy } \\
(n=11)\end{array}$ & $\begin{array}{l}\text { Sum of phakic and } \\
\text { pseudophakic eyes, } \\
\text { only vitrectomy } \\
(n=7+11=18)\end{array}$ \\
\hline BCVA, logMAR & $0.1 \pm 0.11$ & $0.15 \pm 0.17$ & $0.26 \pm 0.22$ & $0.2 \pm 0.2$ \\
CRT, $\mu \mathrm{m}$ & $404.5 \pm 32.2^{* *}$ & $440.6 \pm 51.2$ & $390.4 \pm 71.4^{*}$ & $400.2 \pm 58.3^{*}$ \\
Central CT, $\mu \mathrm{m}$ & $213.9 \pm 34.1$ & $253 \pm 53.6$ & $180.5 \pm 54.3$ & $193.5 \pm 49.3$ \\
Central CV, $\mathrm{mm}^{3}$ & $0.183 \pm 0.04$ & $0.219 \pm 0.04$ & $0.204 \pm 0.04$ & $0.196 \pm 0.034$ \\
\hline
\end{tabular}

BCVA, best-corrected visual acuity; $\mathrm{CT}$, choroidal thickness; $\mathrm{CRT}$, central retinal thickness; $\mathrm{CV}$, choroidal volume; BSL, baseline; $\mathrm{M} 3$, month 3 . Significantly relevant differences in comparison to BSL values are marked: ${ }^{*} p<0.05 ;{ }^{* *} p<0.01$, and ${ }^{* * *} p<0.001$. gery and vitrectomy. Changes of CT and CRT values depending on the lens status and the surgical procedure are shown in Figure 3 and in Tables 3 and 4. Interestingly, functional outcome did not differ between these groups, each showing recovery at M3.

\section{Discussion}

iERM is a fibrocellular proliferation on the retinal surface, resulting in retinal wrinkling and distortion [1-4]. Its pathogenesis is not fully understood and several explanations exist in the literature.

Recent studies have suggested that mechanical traction and ischemia may play a role in idiopathic ERM development. The iERM applies traction on the retina that causes an increase of CT and affects retinal vessels. Retinal arteries become dilated and tortuous in the natural course of ERM formation and as a result may alter the hemodynamics of the affected area $[5-8,18-22]$. The blood flow area of choriocapillaris was reduced in patients with iERM and increased after vitrectomy $[8,9]$. CT was also reduced in the presence of iERM but results differed after surgery $[10,11,23,24]$. Although Li et al. [8] showed that the blood flow area of choriocapillaris increased after vitrectomy, a significant change in CT after vitrectomy was not detected. In contrast, Michalewska et al. $[10,11]$ observed a normalization of CT after vitrectomy and ERM peeling, suggesting that there is some relationship between CT and the presence of ERM. Ahn et al. [24] also showed a temporary increase of CT in the early postoperative period, followed by a decrease of CT 
values after vitreoretinal surgery for ERM and macular hole. However, there is still information lacking on detailed analysis, whether ERM severity could influence the clinical outcome of CT measurements.

Our work focused on studying retinal and CT changes in patients with idiopathic ERM before and after vitreomacular surgery depending on the severity of ERM. For this purpose, patients included in the study were further categorized following the novel OCT staging scheme for ERMs by Govetto et al. [15, 16, 25], in which the presence of ectopic inner foveal layers play an important role in the categorization, as their development is accompanied by a significant drop in visual acuity. Our results were in line with these observations. Furthermore, postoperative improvement of the visual acuity (BCVA) also differed depending on ERM stage, being the largest in stage 3 and 4 ERM, where ectopic inner foveal layers initially occurred. Accordingly, CRT reduction after vitreoretinal surgery was largest in stage 3 and 4 ERMs and showed a strong correlation with $\operatorname{BCVA}\left(r_{2}=0.85\right)$.

We further studied CT changes depending on the severity of ERMs. The thickness of the underlying choroid did not show significant differences among the stages or in comparison to the control eyes. A correlation between BCVA and CT at BSL or in the postoperative period was not observed either. Nevertheless, the anatomical changes of the choroid did not seem to influence the functional outcome of ERM surgery in our study. Therefore, we do hypothesize that the severity of tractional stress exerted by the ERM on the retinal layers does not significantly affect choroidal layers. Our findings correspond with those of Li et al. [8], who also did not detect significant changes in CT after vitrectomy. As proposed by Li et al. [8], different results in CT after vitrectomy might be by reason of differences in factors such as study design, sample size and follow-up period.

A further interesting observation in our patient cohort was made. Depending on the lens status, CT was significantly larger in phakic than in pseudophakic eyes at BSL. After vitrectomy, CT values showed a further reduction in pseudophakic and in phakic eyes without combined surgery. However, if combined phacoemulsification and vitrectomy were performed, CT values remained unchanged and significantly higher over time. In comparison, CRT values did not differ significantly at BSL between phakic and pseudophakic eyes. In the postoperative follow-up, the least reduction of retinal thickness was detected in patients who underwent combined cataract surgery and vitrectomy. Our results do dissent with those of Casini et al. [26], who proposed that the effect of vitrectomy on CT outweighs that induced by cataract surgery. Casini et al. [26] studied CT changes after 25-Gauge vitrectomy for idiopathic ERM with or without cataract surgery and found no statistical differences in CT changes between the 2 groups. In contrast, our results suggest a reduction of $\mathrm{CT}$ in the postoperative period, if vitrectomy without cataract surgery is performed. If cataract surgery and vitrectomy are combined, CT values remain higher postoperatively. A possible explanation for our findings might be inflammatory processes that are caused by cataract surgery. Previous work has shown an increase in subfoveal CT after uncomplicated cataract surgery that was attributed to inflammation. The exact mechanism of this inflammation is not clear and multiple contradictions exist in the literature. One theory implies that postoperative inflammation causes a release of pro-inflammatory prostaglandins and cytokines during surgery, which are also considered to cause macular edema after cataract surgery. Still, such inflammatory responses are expected in other intraocular procedures as well, such as vitrectomy without cataract surgery [26-29]. Our hypothesis is that the effect of cataract surgery on CT might still be, as opposed to the proposition of Casini et al. [26], more significant than that induced by vitreomacular surgery. To confirm this hypothesis, further studies with longer follow-up periods and larger sample sizes are needed. Furthermore, large studies with long follow-up periods on pseudophakic patients are also needed because very scarce data exists regarding the possible long-term effects of cataract surgery on the choroid.

In conclusion, our findings imply that the development of ERM is not related to CT, but a mild thickening of the choroid that does not influence the functional outcome was observed. After vitrectomy, the exposition of ocular tissues to a more highly oxygenated environment may cause a constriction of the choroidal vessels, and CT reduction may occur, but these anatomical changes do not seem to have an influence on the functional outcome of treatment. In the postoperative period, combined surgery seems to cause longer anatomical recovery in retinal and choroidal thickening; however, the functional outcome was independent from the lens status.

\section{Acknowledgements}

We thank the team of the Vienna Clinical Trial Center, especially Maximilian Lindell, for their contribution.
Zobor/Sacu/Hollaus/Aliyeva/Schmalek/ Schmidt-Erfurth/Georgopoulos 


\section{Statement of Ethics}

This study was conducted in adherence to the Declaration of Helsinki. The study protocol was reviewed and approved by the Institutional Ethics Committee of the Medical University of Vienna, Vienna General Hospital, Austria, approval number EK 1020/2014 (www.clinicaltrials.gov, NCT02617147). All patients gave written informed consent.

\section{Conflict of Interest Statement}

The authors have no conflicts of interest to declare.

\section{Funding Sources}

The research for this paper was supported by Department of Ophthalmology, Medical University of Vienna, Vienna General Hospital, Austria. This research did not receive any specific grant from funding agencies in the public, commercial, or not-for-profit sectors.

\section{Author Contributions}

Gergely Zobor was involved in data collection, data analysis, statistical analysis, manuscript, preparation, manuscript approval. Stefan Sacu contributed to study design, clinical examination, manuscript review, manuscript approval. Marlene Hollaus contributed to data analysis, manuscript approval. Leyla Aliyeva contributed data collection, data analysis, manuscript approval. Alexandra-Stephanie Schmalek contributed to data collection, clinical examination, manuscript approval. Ursula-Schmidt-Erfurth contributed to study design, manuscript review, manuscript approval. Michael Georgopoulos contributed to study design, clinical examination, manuscript review, manuscript approval.

\section{Data Availability Statement}

All data generated or analysed during this study are included in this article. Further enquiries can be directed to the corresponding author.

\section{References}

1 Klein R, Klein BE, Wang Q, Moss SE. The epidemiology of epiretinal membranes. Trans Am Ophthalmol Soc. 1994;92:403-30.

2 Mitchell P, Smith W, Chey T, Wang JJ, Chang A. Prevalence and associations of epiretinal membranes. The Blue Mountains Eye Study, Australia. Ophthalmology. 1997; 104:103340.

3 Chang WC, Lin C, Lee CH, Sung TL, Tung $\mathrm{TH}$, Liu JH. Vitrectomy with or without internal limiting membrane peeling for idiopathic epiretinal membrane: a meta-analysis. PLoS One. 2017 Jun 16;12(6):e0179105.

4 Watanabe K, Tsunoda K, Mizuno Y, Akiyama K, Noda T. Outer retinal morphology and visual function in patients with idiopathic epiretinal membrane. JAMA Ophthalmol. 2013 Feb;131(2):172-7.

5 Yoon YS, Woo JM, Woo JE, Min JK. Superficial foveal avascular zone area changes before and after idiopathic epiretinal membrane surgery. Int J Ophthalmol. 2018 Oct 18;11(10): 1711-5.

6 Kadonosono K, Itoh N, Nomura E, Ohno S. Perifoveal microcirculation in eyes with epiretinal membranes. $\mathrm{Br} \mathrm{J}$ Ophthalmol. 1999;83:1329-31.

7 Kono T, Kato H, Oshima K. [Immunohistochemical study of retinal Muller cell response in experimental ERM formation]. Nihon Ganka Gakkai Zasshi. 1998;102:22-7. Japanese.

8 Li Z, Zhang J, Lin T, Peng W, Lu L, Hu J. Macular vascular circulation and retinal oxygen saturation changes for idiopathic macular epiretinal membrane after vitrectomy. Acta Ophthalmol. 2019;97:296-302.
9 Fang I-M, Hsu H-Y, Chiang W-L, Shih Y-L, Han C-L. Correlation between visual acuity and optical coherence tomography angiography parameters in unilateral idiopathic epiretinal membrane. J Clin Med. 2020;10(1): 26.

10 Michalewska Z, Michalewski J, Adelman RA, Zawiślak E, Nawrocki J. Choroidal thickness measured with swept source optical coherence tomography before and after vitrectomy with internal limiting membrane peeling for idiopathic epiretinal membranes. Retina. 2015 Mar;35(3):487-91.

11 Michalewska Z, Michalewski J, Ornafel-Sagan K, Nawrocki J. Swept-source optical coherence tomography correlations between retina and choroid before and after vitrectomy for epiretinal membranes. Am J Ophthalmol. 2016 May; 165:100-7.

12 Spaide RF, Koizumi H, Pozzoni MC, Pozonni MC. Enhanced depth imaging spectral-domain optical coherence tomography. Am J Ophthalmol. 2008;146:496-500.

13 Schlanitz FG, Baumann B, Kundi M, Sacu S, Baratsits M, Scheschy U, et al. Drusen volume development over time and its relevance to the course of age-related macular degeneration. Br J Ophthalmol. 2017;101:198-203.

14 Sayegh RG, Sacu S, Dunavölgyi R, Kroh ME, Roberts P, Mitsch C, et al. Geographic atrophy and foveal-sparing changes related to visual acuity in patients with dry age-related macular degeneration over time. Am J Ophthalmol. 2017;179:118-28.

15 Govetto A, Lalane RA 3rd, Sarraf D, Figueroa MS, Hubschman JP. Insights into epiretinal membranes: presence of ectopic inner foveal layers and a new optical coherence tomography staging scheme. Am J Ophthalmol. 2017 Mar;175:99-113.

16 Govetto A, Bhavsar KV, Virgili G, Gerber MJ Freund $\mathrm{KB}$, Curcio CA, et al. Tractional abnormalities of the central foveal bouquet in epiretinal membranes: clinical spectrum and pathophysiological perspectives. Am J Ophthalmol. 2017 Dec;184:167-80.

17 Wakatsuki Y, Shinojima A, Kawamura A, Yuzawa M. Correlation of aging and segmental choroidal thickness measurement using swept source optical coherence tomography in healthy eyes. PLoS One. 2015 Dec 3;10(12): e0144156.

18 Zhao F, Gandorfer A, Haritoglou C, Scheler $\mathrm{R}$, Schaumberger MM, Kampik A, et al. Epiretinal cell proliferation in macular pucker and vitreomacular traction syndrome: analysis of flat-mounted internal limiting membrane specimens. Retina. 2013;33:77-88.

19 Guidry C, Bradley KM, King JL. Tractional force generation by human Muller cells: growth factor responsiveness and integrin receptor involvement. Invest Ophthalmol Vis Sci. 2003;44:1355-63.

20 Khono RI, Hata Y, Kawahara S, Kita T, Arita $\mathrm{R}$, Mochihuzi Y, et al. Possible contribution of hyalocytes to idiopathic epiretinal membrane formation and its contraction. Br J Ophthalmol. 2009;93:1020-6.

21 Parapuram SK, Chang B, Li L, Hartung RA, Chalam KV, Nair-Menon JU, et al. Differential effects of TGFbeta and vitreous on the transformation of retinal pigment epithelial cells. Invest Ophthalmol Vis Sci. 2009;50: 5965-74. 
22 Sebag J, Gupta P, Rosen RR, Garcia P, Sadun AA. Macular holes and macular pucker: the role of vitreoschisis as imaged by optical coherence tomography/scanning laser ophthalmoscopy. Trans Am Ophthalmol Soc. 2007; 105:121-31.

23 Rizzo S, Savastano A, Finocchio L, Savastano MC, Khandelwal N, Agrawal R. Choroidal vascularity index changes after vitreomacular surgery. Acta Ophthalmol. 2018 Dec;96(8): e950-5.

24 Ahn SJ, Woo SJ, Park KH. Choroidal thickness change following vitrectomy in idiopathic epiretinal membrane and macular hole. Graefes Arch Clin Exp Ophthalmol. 2016; 254:1059-67.
25 Govetto A, Virgili G, Rodriguez FJ, Figueroa MS, Sarraf D, Hubschman JP. Functional and anatomical significance of the ectopic inner foveal layers in eyes with idiopathic epiretinal membranes: surgical results at 12 months. Retina. 2019;39(2):347-57.

26 Casini G, Loiudice P, Lazzeri S, Pellegrini M, Ripandelli G, Figus M, et al. Analysis of choroidal thickness change after 25 -gauge vitrectomy for idiopathic epiretinal membrane with or without phacoemulsification and intraocular lens implantation. Ophthalmologica. $2017 ; 237(2): 78-84$.
27 Pierru A, Carles M, Gastaud P, Baillif S. Measurement of subfoveal choroidal thickness after cataract surgery in enhanced depth imaging optical coherence tomography. Invest Ophthalmol Vis Sci. 2014;55(55):4967-74.

28 Noda Y, Ogawa A, Toyama T, Ueta T. Longterm increase in subfoveal choroidal thickness after surgery for senile cataracts. Am J Ophthalmol. 2014;158:455-9.e1.

$29 \mathrm{Xu} \mathrm{H}$, Chen M, Forrester JV, Lois N. Cataract surgery induces retinal pro-inflammatory gene expression and protein secretion. Invest Ophthalmol Vis Sci. 2011;52(1):249-55. 